\title{
Profiling of Protein Degraders in Cultures of Human Gut Microbiota
}

\begin{abstract}
Alberto Amaretti',2, Caterina Gozzoli', Marta Simone', Stefano Raimondi', Lucia Righini ${ }^{1}$, Vicente Pérez-Brocal ${ }^{3,4}$, Rodrigo García-López ${ }^{3,4}$, Andrés Moya ${ }^{3,4,5}$ and Maddalena Rossi ${ }^{1,2 *}$

${ }^{1}$ Department of Life Sciences, University of Modena and Reggio Emilia, Modena, Italy, ${ }^{2}$ BIOGEST-SITEIA, University of Modena and Reggio Emilia, Modena, Italy, ${ }^{3}$ Area of Genomics and Health, Foundation for the Promotion of Sanitary and Biomedical Research of Valencian Community (FISABIO-Public Health), Valencia, Spain, ${ }^{4}$ CIBER in Epidemiology and Public Health (CIBERESP), Madrid, Spain, ${ }^{5}$ Institute for Integrative Systems Biology (I ${ }^{2}$ SysBio), University of Valencia, Spanish National Research Council (CSIC-UVEG), Valencia, Spain
\end{abstract}

Unabsorbed proteins reach the colon and are fermented by the microbiota, yielding a variety of harmful metabolites. In the present study, a 16S rRNA gene survey identified the bacterial taxa flourishing in 11 batch fermentations with proteins and peptones as the sole fermentable substrates, inoculated with the feces of six healthy adults. Organic acids, ammonia, and indole resulting from protein breakdown and fermentation accumulated in all of the cultures. Analysis of differential abundances among time-points identified Enterobacteriaceae, Burkholderiaceae, and Desulfovibrionaceae (including Esherichia-Shigella, Sutterella, Parasutterella, and Bilophila) among the bacteria that especially in the cultures with low inoculation load. Lachnospiraceae and Ruminococcaceae also encompassed many taxa that significantly expanded, mainly in cultures inoculated with high inoculation load, and showed the strongest correlation with the production of ammonium, indole, and p-cresol. Anaerotruncus, Dorea, Oscillibacter, Eubacterium oxidoreducens, Lachnoclostridium, Paeniclostridium, and Rombutsia were among them. Other Firmicutes (e.g., Roseburia, Ruminococcus, Lachnospira, Dialister, Erysipelotrichaceae, and Streptococcaceae) and many Bacteroidetes (e.g., Barnesiellaceae, Prevotellaceae, and Rickenelliaceae) decreased. Sequences attributed to Bacteroides, unresolved at the level of species, presented opposite contributions, resulting in no significant changes in the genus. This study sheds light on the multitude of bacterial taxa putatively participating in protein catabolism in the colon. Protein fermentation was confirmed as unfavorable to health, due to both the production of toxic metabolites and the blooming of opportunistic pathogens and pro-inflammatory bacteria.

Keywords: gut microbiota, proteolysis, metagenomics, enrichment culture, Enterobacteriaceae

\section{INTRODUCTION}

The human colonic microbiota is a dense and complex community of commensal microbes, mostly bacteria, gaining energy and nourishment in strict anaerobiosis from non-digestible dietary substrates and host-derived secretions (Scott et al., 2013). Non-digestible oligo- and polysaccharides are the substrate of intestinal saccharolytic bacteria. Carbohydrate fermentation 
mainly yields short-chain fatty acids (SCFA), which fuel the enterocytes and are major contributors to the maintenance of gut function and to immune homeostasis (Sivaprakasam et al., 2016). Therefore, intestinal saccharolytic catabolism is regarded as beneficial for the maintenance of health and has attracted considerable attention in the last few decades, leading to the development of prebiotic supplements (Gänzle and Follador, 2012; Wilson and Whelan, 2017).

Proteins are another major source of carbon and energy for colonic bacteria. Though most dietary proteins are digested and absorbed in the small intestine, a load of unabsorbed proteins and peptides reaches the colon, where it serves as a substrate for fermentation by the resident bacteria. In the colon, protein breakdown takes advantage of the host's endopeptidases and bacterial proteases (Cummings and Macfarlane, 1991). The proteolysis yields peptides and/or amino acids that can be subjected to Stickland reactions or other fermentation pathways by amino acid-fermenting bacteria (Smith and Macfarlane, 1998; Kim et al., 2004). Carbon dioxide, ammonia, and organic acids bearing the side chain of the amino acids are the primary products of amino acid fermentation (Barker, 1981; Riedel et al., 2017). The organic acids can further serve as electron acceptors or donors in the oxidation or reduction of other compounds (keto-acids, $\mathrm{H}_{2}$, unsaturated fatty acids) and can undergo decarboxylation and transamination reactions (Davila et al., 2013). Therefore, the range of fermentation products encompasses linear and branched organic acids (acetate, butyrate, propionate, valerate, isobutyrate, 2-methylbutyrate, isolavalerate, etc.), phenols and indole derivatives originating from aromatic amino acids, and a variety of nitrogen or sulfur-containing compounds (Smith and Macfarlane, 1996; Davila et al., 2013; Russell et al., 2013a). Unlike the saccharolytic catabolism, many of these products are undisputedly detrimental to health (in particular ammonia, phenols, indoles, amines, sulfides, and $N$-nitroso compounds), having a variety of harmful effects, including systemic toxicity, nephrotoxicity, and carcinogenesis (Blachier et al., 2010; Russell et al., 2013a,b; Barrios et al., 2015; Kobayashi, 2017).

A large amount of information on the human gut microbiota has been generated in recent years, favored by next-generation sequencing (NGS) technologies (Moore-Connors et al., 2016). NGS enabled and drove the detailed description of microbiota composition but the assessment of the functional role of bacteria was generally neglected. In recent years, many studies have been done on the effects of dietary protein on gut microbiota ( $\mathrm{Wu}$ et al., 2011; David et al., 2014; Ma et al., 2017); nevertheless, most functional information on the bacteria involved in gut protein catabolism come from culture-based studies dependent on the isolation and physiological characterization of cultivable species (Cummings and Macfarlane, 1991; Smith and Macfarlane, 1998; Russell et al., 2013a). Clostridia and peptostreptococci are the most frequent isolates in media containing amino acids as energy and carbon sources, along with a wide range of bacteria belonging to Fusobacterium, Bacteroides, Propionibacterium, Actinomyces, Enterobacteria, and Gram-positive cocci (Davila et al., 2013). In the present study, fecal batch cultures with a medium containing only proteins and peptones as fermentable carbon sources were carried out in order to enrich the bacterial fraction taking advantage of protein fermentation. The progression of the microbiota was monitored by $16 \mathrm{~S}$ rRNA gene profiling in order to identify the taxa involved in protein breakdown and fermentation.

\section{MATERIALS AND METHODS}

\section{Chemicals and Culture Medium}

All the chemicals were purchased from Sigma-Aldrich (Steinheim, Germany) unless otherwise stated. The Protein Fermenter Medium (PFM) medium, developed by modifying Walker et al.'s (2005), contained $1.5 \mathrm{~g} / \mathrm{L}$ sodium caseinate, $1.5 \mathrm{~g} / \mathrm{L}$ beef extract, $1 \mathrm{~g} / \mathrm{L}$ peptone (BD Difco, Sparks, NV, United States), $2 \mathrm{~g} / \mathrm{L} \mathrm{KH}_{2} \mathrm{PO}_{4}, 4.5 \mathrm{~g} / \mathrm{L} \mathrm{NaCl}, 0.5 \mathrm{~g} / \mathrm{L} \mathrm{MgSO}_{4}$. $7 \mathrm{H}_{2} \mathrm{O}, 0.045 \mathrm{~g} / \mathrm{L} \mathrm{CaCl} \cdot 2 \mathrm{H}_{2} \mathrm{O}, 0.005 \mathrm{~g} / \mathrm{L} \mathrm{FeSO}_{4} \cdot 7 \mathrm{H}_{2} \mathrm{O}, 0.01 \mathrm{~g} / \mathrm{L}$ hemin, $0.05 \mathrm{~g} / \mathrm{L}$ bile salts (Oxgall, BD Difco), $0.6 \mathrm{mg} / \mathrm{L}$ resazurin, and $0.2 \mathrm{~mL} / \mathrm{L}$ antifoam (Xiameter 1520, Dow Corning, Midland, MI, United States). The following filter-sterilized solutions were added to the autoclaved medium: $2 \mathrm{~mL} / \mathrm{L}$ minerals $(500 \mathrm{mg} / \mathrm{L}$ EDTA, $200 \mathrm{mg} / \mathrm{L} \mathrm{FeSO}_{4} \cdot 7 \mathrm{H}_{2} \mathrm{O}, 10 \mathrm{mg} / \mathrm{L} \mathrm{ZnSO}_{4} \cdot 7 \mathrm{H}_{2} \mathrm{O}$, $3 \mathrm{mg} / \mathrm{L} \mathrm{MnCl}_{2} \cdot 7 \mathrm{H}_{2} \mathrm{O} ; 30 \mathrm{mg} / \mathrm{L} \mathrm{H}_{3} \mathrm{BO}_{3}, 20 \mathrm{mg} / \mathrm{L} \mathrm{CoCl}_{2}$. $6 \mathrm{H}_{2} \mathrm{O}, 1 \mathrm{mg} / \mathrm{L} \mathrm{CuCl}_{2} \cdot 2 \mathrm{H}_{2} \mathrm{O}, 2 \mathrm{mg} / \mathrm{L} \mathrm{NiCl} 2 \cdot 6 \mathrm{H}_{2} \mathrm{O}$, and $3 \mathrm{mg} / \mathrm{L} \mathrm{NaMoO}_{4} \cdot 2 \mathrm{H}_{2} \mathrm{O}$ ), $1.4 \mathrm{~mL} / \mathrm{L}$ vitamins ( $1 \mathrm{~g} / \mathrm{L}$ menadione, $2 \mathrm{~g} / \mathrm{L}$ biotin, $2 \mathrm{~g} / \mathrm{L}$ calcium pantothenate, $10 \mathrm{~g} / \mathrm{L}$ nicotinamide, $0.5 \mathrm{~g} / \mathrm{L}$ cyanocobalamin, $0.5 \mathrm{~g} / \mathrm{L}$ folic acid, $4 \mathrm{~g} / \mathrm{L}$ thiamine, and $5 \mathrm{~g} / \mathrm{L}$ PABA), and $40 \mathrm{~mL} / \mathrm{L}$ reducing solution (12.5 g/L L-cysteine . $\mathrm{HCl}$ and $80 \mathrm{~g} / \mathrm{L} \mathrm{NaHCO}_{3}$ ).

\section{Fermentation Experiments}

Fecal samples from six healthy volunteers (three men and three women aged 25-50 years) were collected fresh, having obtained written informed consent, according to the experimental protocol that was approved with ref. no. $125-15$ by the local research ethics committee (Comitato Etico Provinciale, Azienda Policlinico di Modena, Italy). Subjects had not taken prebiotics and/or probiotics for the previous 2 weeks or antibiotics for at least 3 months prior to sample collection. The feces were homogenized 5\% (w/v) with sterile PFM medium under an $85 \% \mathrm{~N}_{2}, 10 \% \mathrm{CO}_{2}, 5 \% \mathrm{H}_{2}$ atmosphere in an anaerobic cabinet (Anaerobic System, Forma Scientific, Marietta, OH). 60 or $3 \mathrm{~mL}$ of the suspension were utilized to seed PFM cultures with a concentrated or a diluted inoculation, 1 or $0.05 \%$ feces $(\mathrm{w} / \mathrm{v})$, respectively. Hereinafter, the fermentations that were inoculated at $1 \%$ are referred to as $\mathrm{C}$ (i.e., concentrated) processes, while the ones inoculated at $0.05 \%$ are referred to as $\mathrm{D}$ (i.e., diluted) processes.

Batch cultures with a volume of $400 \mathrm{~mL}$ were carried out in a multivessel bioreactor apparatus (Sixfors V3.01, Infors, Bottmingen, Switzerland), incubated at $37^{\circ} \mathrm{C}$ under a $\mathrm{CO}_{2}$ atmosphere (Bottari et al., 2017). The $\mathrm{pH}$ was maintained at 6.8 through automatic titration with $1 \mathrm{M} \mathrm{HCl}$ and $1 \mathrm{M} \mathrm{NaOH}$. Samples for $16 \mathrm{~S}$ rRNA gene analysis were collected at 0,6 , and $12 \mathrm{~h}$ of incubation.

A single preliminary fermentation run was carried out with subject V1, seeded with a C inoculation. Parallel fermentation 
runs were carried out for subject V2, both seeded with a $\mathrm{C}$ inoculation. For subjects V3, V4, V5, and V6, parallel fermentation runs were carried out with $\mathrm{C}$ and $\mathrm{D}$ inoculation conditions.

\section{DNA Extraction, 16S rRNA Gene Amplification, Library Construction, and Sequencing}

Total DNA was extracted using a QIAamp DNA Stool Mini Kit (Quiagen, Hilden, Germany) following the manufacturer's protocol. The DNA was quantified with a Qubit 3.0 fluorimeter (Thermo Fisher Scientific, Waltham, MA, United States) in order to normalize the template for $16 \mathrm{~S} \mathrm{rDNA}$ sequencing to $5 \mathrm{ng} / \mu \mathrm{L}$.

Amplicons of approx. $460 \mathrm{bp}$ were generated with PCR primers, targeting the V3 to V4 regions of the $16 \mathrm{~S}$ rRNA gene and including Illumina adapter sequences (F-5'TCGTCGGCAGCGTCAGATGTGTATAAGAGACAGCCT ACGGGNGGCWGCAG, R-5'GTCTCGTGGGCTCGGAGATG TGTATAAGAGACAGGACTACHVGGGTATCTAATCC). The PCR reaction was performed in a 96-well Mastercycler-pro apparatus (Eppendorf, Hamburg, Germany) with the following program: $3 \mathrm{~min}$ at $95^{\circ} \mathrm{C} ; 25 \mathrm{cycles}$ of $30 \mathrm{~s}$ at $95^{\circ} \mathrm{C}, 30 \mathrm{~s}$ at $55^{\circ} \mathrm{C}$, and $30 \mathrm{~s}$ at $72^{\circ} \mathrm{C} ; 72^{\circ} \mathrm{C}$ for $7 \mathrm{~min}$. An Illumina Nextera $\mathrm{XT}$ Index kit (Illumina Inc., San Diego, CA, United States) with dual 8-base indexes was used for multiplexing. The indexes were incorporated into the amplicons by means of $\mathrm{PCR}$ reactions with the following conditions: $3 \mathrm{~min}$ at $95^{\circ} \mathrm{C} ; 25$ cycles of $30 \mathrm{~s}$ at $95^{\circ} \mathrm{C}$, $30 \mathrm{~s}$ at $55^{\circ} \mathrm{C}$, and $30 \mathrm{~s}$ at $72^{\circ} \mathrm{C} ; 72^{\circ} \mathrm{C}$ for $7 \mathrm{~min}$. Both amplification reactions were followed by purification with AMPure XP beads (Beckman-Coulter, Brea, CA, United States).

The amplicons were quantified using the Qubit 3.0 fluorimeter, pooled at a concentration of $4 \mathrm{nM}$ each, and sequenced by $2 \times 300$ bp paired-end sequencing on the MiSeq platform using a MiSeq v3 Reagent Kit (Illumina). Each run included a minimum of 5\% PhiX DNA as an internal control.

\section{Sequence Analysis}

Raw sequences were cleaned and filtered by size and quality using MOTHUR v.1.25.0 software (Schloss et al., 2009). Quality filtered sequences were processed with the QIIME2 pipeline (Version 2019.1) for closed-reference picking of amplicon sequence variants (ASVs), taxonomy assignment, collapsing into operational taxonomic units (OTUs), and diversity metrics computation (Bolyen et al., 2018). Closed-reference picking and taxonomic assignation were carried out utilizing SILVA SSU database release $132^{1}$ as a reference with the similarity threshold set at 0.97 .

Alpha diversity indexes (number of observed taxa, Chao1, Shannon, and Pielou) were computed to evaluate within-sample richness and evenness at different taxonomic levels. KruskalWallis test by ranks was carried out with the appropriate QIIME2 plugin for alpha diversity comparisons between groups $(0,6$, and $12 \mathrm{~h}$ samples and $\mathrm{C}$ and $\mathrm{D}$ cultures), utilizing feature tables rarefied at the same number of reads.

Pairwise sample dissimilarity (beta diversity) was computed with Jaccard, Bray-Curtis, Canberra, Unweighted Normalized

\footnotetext{
${ }^{1}$ https://www.arb-silva.de/download/arb-files/
}

UniFrac, and Weighted Normalized UniFrac metrics resulting in distance/dissimilarity matrices, which enabled the hierarchical clustering of samples in UPGMA trees and Principal Coordinate Analysis (PCoA). Beta diversity comparisons within and between groups were carried through the analysis of similarities (ANOSIM) and permutational multivariate analysis of variance (PERMANOVA), using the appropriate QIIME2 plugin. The Linear discriminant analysis Effect Size (LEfSe) ${ }^{2}$ algorithm was applied to discover distinctive features at different time-points and to compare $\mathrm{C}$ and $\mathrm{D}$ cultures with the founding microbiota (Segata et al., 2011).

Spearman's correlation was computed within the OTUs and between them and the concentration of ammonium, indole, and p-cresol. Before computing the correlation, sparsity reduction was applied on the feature table, keeping only the OTUs with at least 11 total reads (quantile 30\%) and appearing in least in seven samples. The dataset was then rarefied using the smallest sample size. Sparsity reduction, rarefaction, and calculation of correlation were carried out with $\mathrm{R}$ software (version 3.6), with libraries "vegan" and "corrr."

\section{Chemical Analysis}

Acetate, lactate, propionate, butyrate, formate, succinate, and ethanol in the culture supernatants were quantified by HPLC with a refractive index detector (1200 System, Agilent Technologies, Waldbronn, Germany) and an Aminex HPX-87 H ion exclusion column. Isocratic elution was carried out at $60^{\circ} \mathrm{C}$ with $0.8 \mathrm{~mL} / \mathrm{min}$ of $5 \mathrm{mM} \mathrm{H}_{2} \mathrm{SO}_{4}$ (Amaretti et al., 2013).

Indole and $p$-cresol were quantified by an HPLC (1100 System, Agilent Technologies) equipped with a diode array detector and a C18 Kinetex column (Phenomenex, Torrance, CA, United States). Elution was carried out at the flow of $0.8 \mathrm{~mL} / \mathrm{min}$, with a gradient of $0.1 \%(\mathrm{v} / \mathrm{v})$ formic acid in water (eluent $\mathrm{A})$ and $0.1 \%(\mathrm{v} / \mathrm{v})$ formic acid in methanol (eluent B), eluent B increasing from 30 to $95 \%$ in $15 \mathrm{~min}$ and remaining at $95 \%$ till $20 \mathrm{~min}$. Detection was carried out at $275 \mathrm{~nm}$.

Ammonium was analyzed with a colorimetric assay. Briefly, $400 \mu \mathrm{L}$ of properly diluted supernatant was mixed with $400 \mu \mathrm{L}$ of Phenol nitroprusside solution (Sigma-Aldrich, P6994) and $400 \mu \mathrm{L}$ of Alkaline Hypochlorite solution (Sigma-Aldrich, A1727) and incubated at room temperature for $30 \mathrm{~min}$. Absorbance was read at $625 \mathrm{~nm}$ and compared with a calibration curve in the range from 0 to $150 \mu \mathrm{M}$.

Total carbohydrates were analyzed with anthrone colorimetric assay. $500 \mu \mathrm{L}$ of culture was mixed with $1,000 \mu \mathrm{L}$ of $2 \mathrm{~g} / \mathrm{L}$ anthrone in $\mathrm{H}_{2} \mathrm{SO}_{4} 96 \%$, incubated at $10 \mathrm{~min}$ at $100^{\circ} \mathrm{C}$, then cooled in ice. Absorbance was measured at $620 \mathrm{~nm}$ and compared with a calibration curve in the range from 0 to $150 \mathrm{mg} / \mathrm{L}$.

ANOVA followed by Tukey's post hoc test was carried out to analyze the trend of metabolites over time in C and D fermentation, separately. Differences were considered statistically significant for $P<0.05$. The $t$-test was utilized to compare the means of metabolite concentrations in $\mathrm{C}$ and $\mathrm{D}$ cultures at each time-point. Differences were considered statistically significant

\footnotetext{
${ }^{2}$ http://huttenhower.sph.harvard.edu/galaxy
} 
for $P<0.05$. The statistical analysis was performed with IBM SPSS Statistics 21 software.

\section{RESULTS}

\section{Batch Cultures of Human Gut Microbiota in Protein-Based Medium}

The time course of the concentration of metabolites in C and D cultures (mean) is reported in Figure 1, with single runs shown in Supplementary Figure S1. Ammonium was produced during all the fermentations. In $\mathrm{C}$ processes, it reached a stationary concentration of $13.0 \pm 1.1 \mathrm{mM}$ (mean $\pm \mathrm{SD}$ ) at $24 \mathrm{~h}$, with most of the production (at least $75 \%$ of the final concentration) occurring in the first $12 \mathrm{~h}$. Ammonium production was slower in $\mathrm{D}$ processes, which yielded a lower concentration at $24 \mathrm{~h}$ $(8.7 \pm 0.9 \mathrm{mM})$ compared to $C$ processes $(P<0.05)$, and were protracted throughout the whole course of fermentation. Similar behavior was observed for indole, which reached a maximum of $63 \pm 23 \mu \mathrm{M}$ (mean $\pm \mathrm{SD}$ ) at $12 \mathrm{~h}$ in $\mathrm{C}$ fermentations but was produced more slowly in D runs, yielding $39 \pm 22 \mu \mathrm{M}$ at the same time-point $(P<0.05)$. Unlike ammonium and indole, $p$-cresol was produced mostly toward the end of the fermentation, being always more abundant in $\mathrm{C}$ than in $\mathrm{D}$ runs $(P<0.05) . p$-cresol was notably abundant in V2 processes, where it reached $40 \mu \mathrm{M}$ at the end of the fermentation (Supplementary Figure S1).

Acetic, propionic, and butyric acids were the main organic acids that accumulated throughout the fermentations. In $\mathrm{C}$ fermentations, they had all already reached a stationary level at 6 or $12 \mathrm{~h}$, while production was slower in D processes and reached stationary values at 12 or $24 \mathrm{~h}$. In C fermentations, acetic, propionic, and butyric acids reached $0.85 \pm 0.29,0.28 \pm 0.14$, and $0.28 \pm 0.08 \mathrm{~g} / \mathrm{L}$ (mean $\pm \mathrm{SD}$ ) after $12 \mathrm{~h}$, while significantly lower concentrations $(P<0.05)$ were observed in $\mathrm{D}$ fermentations $(0.33 \pm 0.11,0.12 \pm 0.05,0.13 \pm 0.05 \mathrm{~g} / \mathrm{L}$, respectively). Succinic and formic acids were also produced, although in low amounts. They transiently accumulated in the first $12 \mathrm{~h}$, never increasing above $0.12 \mathrm{~g} / \mathrm{L}$, then decreased to negligible concentrations toward the end of the fermentation. Net ethanol production started at $12 \mathrm{~h}$ and continued to the end of fermentation, reaching up to $0.2 \pm 0.1 \mathrm{~g} / \mathrm{L}$ (mean $\pm \mathrm{SD}$ ). With regard to succinic acid, formic acid, and ethanol, no significant differences were observed between $\mathrm{C}$ and $\mathrm{D}$ processes $(P>0.05)$. Carbohydrates were always $<80 \mathrm{mg} / \mathrm{L}$ at $0 \mathrm{~h}$ and were depleted in the first $6 \mathrm{~h}$.

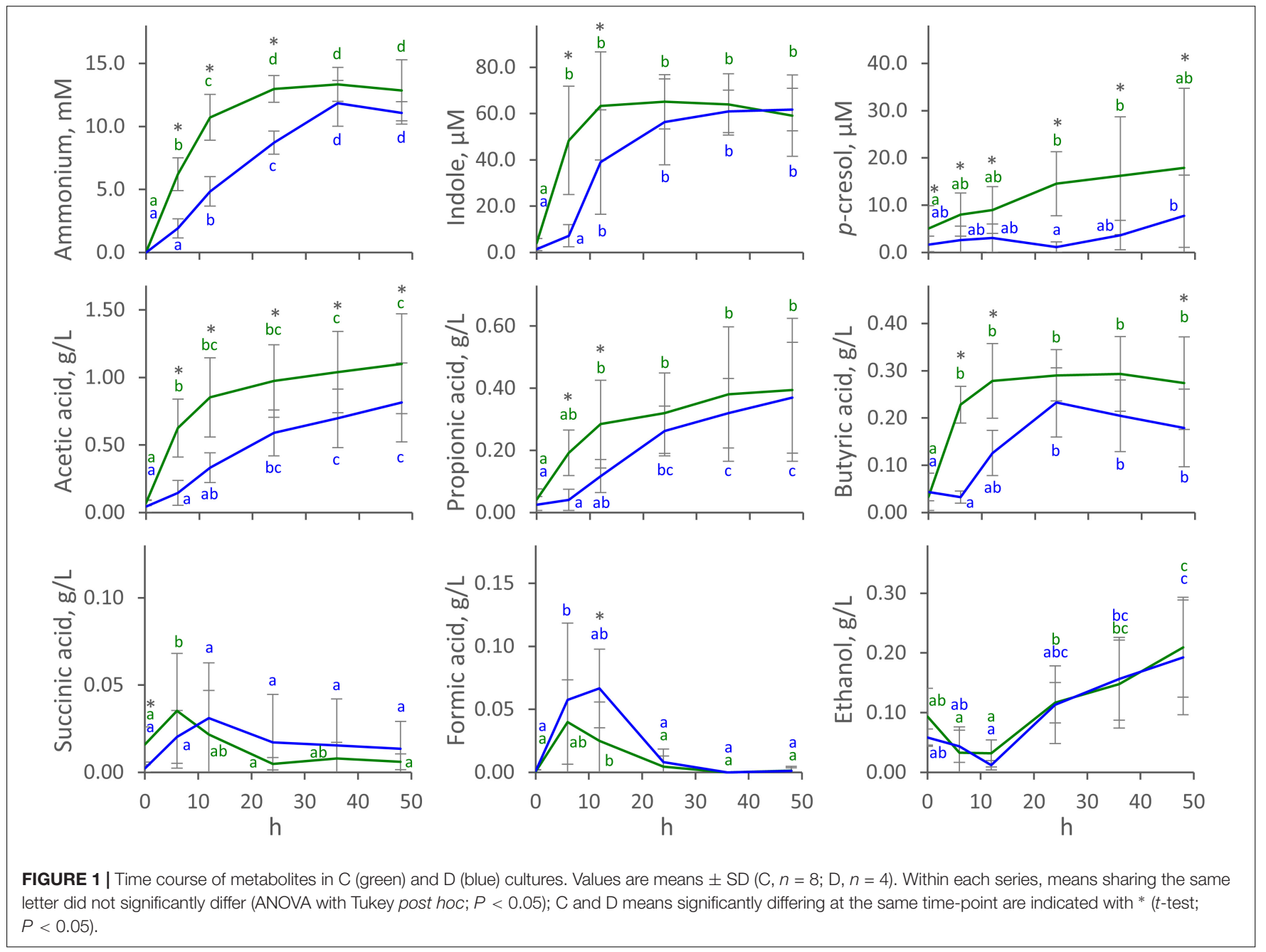



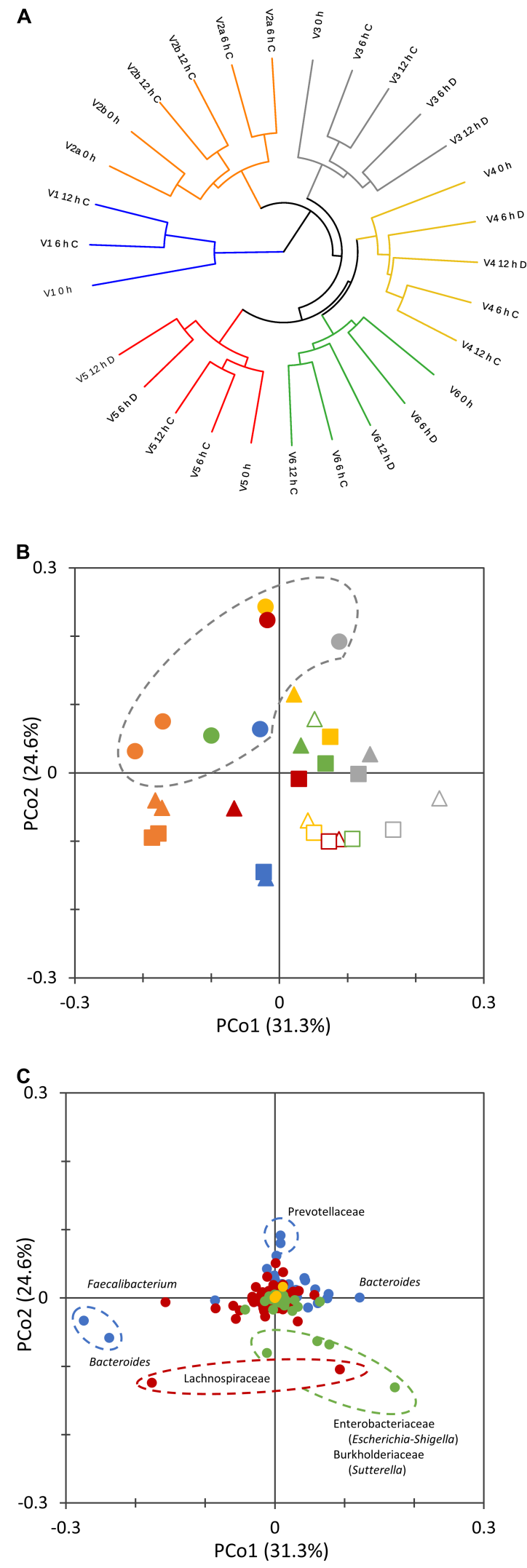

FIGURE 2 | Continued
FIGURE 2 | Beta diversity analysis of microbiota cultures at different times during cultivation. UPGMA dendrogram displaying Bray-Curtis distances, computed with the taxonomic features collapsed the level of species (A). 2D PCoA visualization of phylogenetic distances, computed as Normalized Weighted Unifrac (B,C). Panel (B) reports the scores of samples from different subjects (V1, cyan; V2, orange; V3, gray; V4, yellow; V5, red; V6, green) at 0 (circle), 6 (triangle), and $12 \mathrm{~h}$ (square) of cultivation. Empty symbols correspond to D cultures. Panel $\mathbf{( C )}$ reports the contribution of single bacterial taxa. Bacteroidetes, blue; Firmicutes, red; Proteobacteria, green;

Verrucomicrobia, yellow; other phyla, gray.

\section{Diversity of Microbiota Cultures}

The 16S rRNA gene profile of the cultures was determined at 0,6 , and $12 \mathrm{~h}$, when most of the protein fermentation was observed. A total of 1,685,682 quality-trimmed $16 \mathrm{~S}$ rRNA gene sequences were obtained from 29 samples, on average 58,127 reads per sample (Supplementary Datasheet S1). Sequences were dereplicated into $4,174 \mathrm{ASV}$, hitting a reference sequence in the Silva database, and collapsed at the seventh level of taxonomic annotation (i.e., the species, if available) into 411 OTUs.

Richness (number of OTUs and Chaol index) was higher at $0 \mathrm{~h}$ than at 6 and $12 \mathrm{~h}(P<0.05)$, while the evenness (Shannon and Pielou indexes) did not change during the process $(P>0.05)$ (Supplementary Figure S2). Regardless of the incubation time, both the richness and the evenness were significantly higher in C than in $\mathrm{D}$ processes.

The non-phylogenetic beta diversity metrics evidenced subject-based clusters, with samples not grouping according to the time or the concentration of the inoculum (Figure 2A). With UniFrac metrics, which consider phylogenetic distances, groups based on the incubation time $(0,6$, and $12 \mathrm{~h})$ could be distinguished (Figure 2B). Time-based groups had different centroids (PERMANOVA, $P=0.002$ ), and the similarity within each group was significantly higher than that between them (ANOSIM, $P=0.002$ ). $0 \mathrm{~h}$ samples lay in the same region in the PCoA plot and similarly moved toward low PCo1 and high PCo2 values during the fermentation.

The twin processes seeded with the same amount of microbiota from subject V2 behaved similarly. Within the whole set of cultures, the distance between samples at 0 and $6 \mathrm{~h}$ was generally higher than that between 6 and $12 \mathrm{~h}$. For the pairs of processes that were inoculated with different amounts of microbiota, $\mathrm{D}$ cultures evolved more distantly from the initial microbiota than did C cultures $(P<0.05)$.

The ASVs that mainly contributed to negative $\mathrm{PCo} 2$ belonged to the Enterobacteriaceae (Escherichia-Shigella), Burkholderiaceae (Sutterella), and Lachnospiraceae families, whereas Prevotellaceae were the main drivers of positive PCo2 (Figure 2C). Sequences ascribed to the genus Bacteroides highly contributed to both positive and negative PCo1, which was negatively driven also by Faecalibacterium.

\section{Microbiota Evolution and Analysis of Differential Abundances}

The evolution of microbiota is reported in Figure 3. A total of 64 taxa exhibited significant difference between 0 and $12 \mathrm{~h}$ 




FIGURE 3 | Stacked bar-plot representation of microbiota compositions during C and D batch cultivation, with taxonomic features collapsed at the level of phyla (A) families (B), and genera (C). The phyla, families, and genera that remained unclassified or never occurred with abundance higher than $2.5 \%$ are grouped as others $(*)$.

(Figure 4), 17 becoming enriched and 47 decreasing. In most cases, significant enrichment was already observed after $6 \mathrm{~h}$. Proteobacteria and taxa ascribed to Ruminococcaceae and
Lachnospiraceae were the main biomarkers of the grown cultures, regardless of inoculation load. Comparison between $\mathrm{C}$ and $\mathrm{D}$ cultures revealed that Firmicutes characterized 

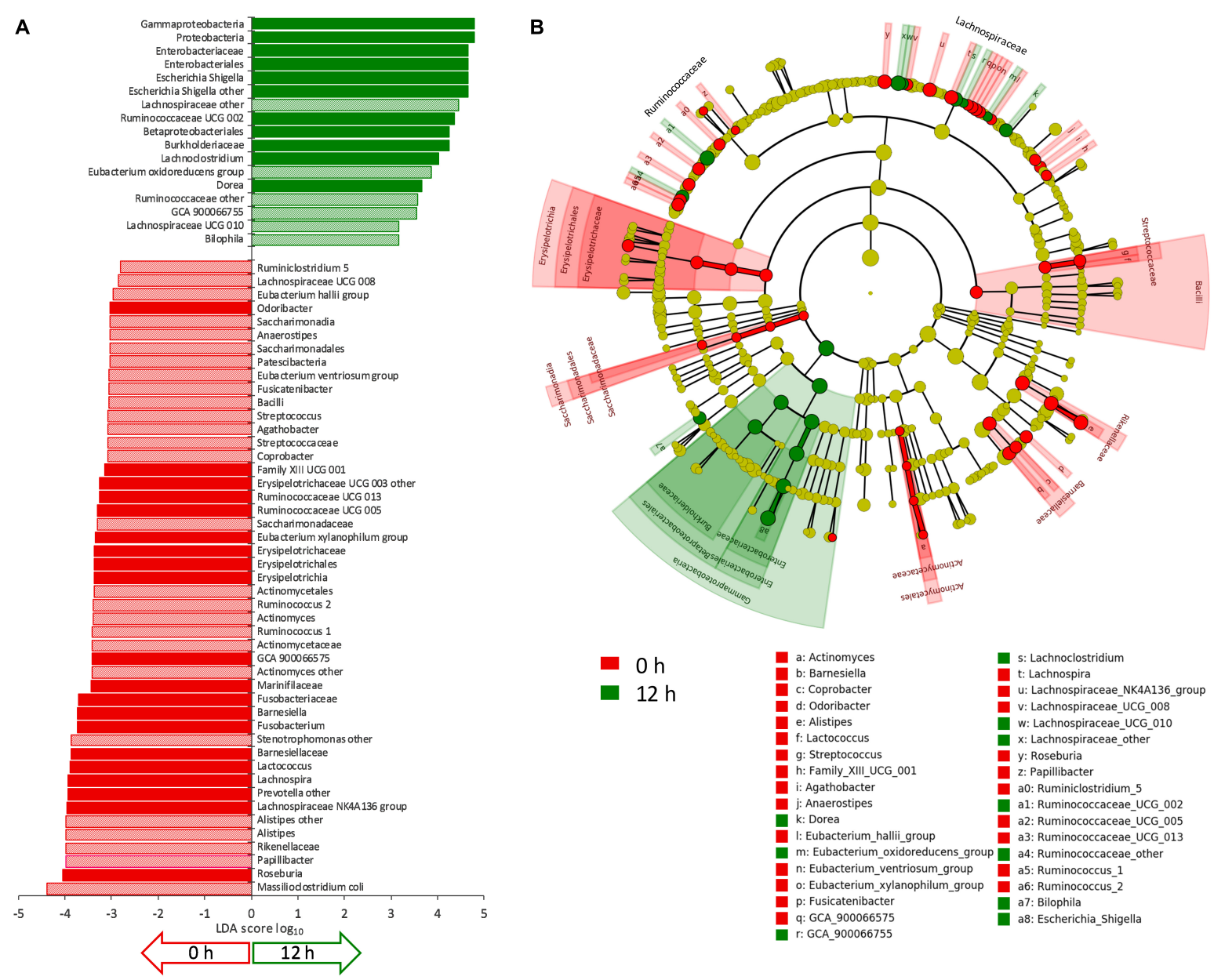

FIGURE 4 | (A) LDA logarithmic scores of taxonomic biomarkers found by LEfSe exhibiting significant differential abundance ( $P<0.05$, logarithmic LDA score $\geq 2.0)$ between 0 and $12 \mathrm{~h}$, regardless of inoculation load ( $\mathrm{C}$ and $\mathrm{D}$ processes). Filled bars represent the taxa with significant differential abundance already after $6 \mathrm{~h}$.

(B) Cladogram visualization of the taxonomic biomarkers characterizing 0 and $12 \mathrm{~h}$.

the former and Proteobacteria the latter (Supplementary Figure S3). The whole phylum Proteobacteria, the class Gammaproteobacteria, and the orders Enterobacteriales (with Enterobacteriaceae) and Betaproteobacteriales (with Burkholderiaceae) significantly increased regardless of the inoculum load but became significantly more abundant in $\mathrm{D}$ cultures than in $\mathrm{C}$ cultures. At the level of genus and species, the Enterobacteriaceae Escherichia-Shigella and the Desulfovibrionaceae Bilophila always characterized the grown cultures, while Xanthomonadales, such as Dyella and Stenotrophomonas maltophila, presented a significant increment only in D cultures. Within Firmicutes, some Lachnospiraceae and Ruminococcaceae (such as Dorea, Eubacterium oxidoreducens, Lachnoclostridium, and other uncultured/unclassified members) were among the most significantly enriched taxa. Their increase was significant regardless of inoculum dilution, but they became more abundant in $\mathrm{C}$ cultures. In particular, the increase of the Lachnospiraceae Sellimonas was observed only in C cultures, together with the Ruminococcaceae Anaerotruncus and Oscillibacter. Within Actinobacteria, Coriobacteriaceae and particularly Collinsella presented significant increments, but only in D cultures.

Other taxa showed a drastic increase and reached remarkably high levels but were not identified as biomarkers by LEfSe because they occurred only in a few founding microbiota or because they thrived only in some of the cultures where they were initially present. Numerous taxa of Proteobacteria increased during the incubation, in some cases starting from values initially lying below the limit of detection, but were detected in a minority of samples. For example, Sutterella was abundant in cultures $\mathrm{V} 1, \mathrm{~V} 3$, and V4, where it increased, particularly in D processes, while Parasutterella was abundant and increased in V2, V5, and V6 cultures. Peptostreptococcaceae were initially negligible in all the cultures and did not present any significant change. Interestingly, they became abundant in the D cultures of V5, where they increased by two magnitudes to $6.3 \%$, mainly because of the contribution of Paeniclostridium and Romboutsia. Among Clostridiaceae, only Clostridium sensu stricto 1, generally absent or negligible at $0 \mathrm{~h}$, became enriched in D processes inoculated with the feces of subject V5 by more than two magnitudes, 
reaching 5.3\%. Acidaminococcus (fam. Acidaminococcaceae) was absent in most of the cultures and abundant only in V5, where it grew considerably. The Verrucomicrobia Akkermansia muciniphila was initially absent or scarce in all of the cultures, and it reached a remarkably high abundance only in V4 cultures, in particular $\mathrm{C}$, where it became one of the dominant taxa $(7.8 \%)$.

Other taxa did not respond positively to proteinaceous substrates. Bacteroidaceae, almost coinciding with the genus Bacteroides, did not significantly change, while other Bacteroidetes, such as Rikenellaceae (including the genus Alistipes), Barnesiellaceae (such as Barnesiella and Coprobacter), and Marinifilaceae (including the genus Odoribacter) significantly decreased. Prevotellaceae were initially remarkably abundant only in V3 and V4 and drastically decreased. Among Firmicutes, many members of Rumicococcaceae and Lachnospiraceae (such a Papillibacter, Ruminiclostridium, Ruminococcus, Subdoligranulum, Agathobacter, Anaerostipes, Fusicatenibacter, Lachnospira, Roseburia, Eubacterium xylanophilum, Eubacterium ventriosum, Eubacterium hallii, and other unclassified members) significantly decreased during cultivation or presented a tendency to decrease (Butyrivibrio). Also Bacilli (such as Streptococcaceae, Streptococcus, and Lactococcus) and Erysipelotrichaceae significantly diminished, while Acidaminococcaceae (Dialister), Christensennellaceae, and Vellionellaceae presented a negative tendency, in particular in the cultures where their relative abundance was initially remarkably high (Dialister in V4; Christensennellaceae in V1 and V6; Vellionellaceae in V1, V4, and V5). The abundance of the saccharolytic bifidobacteriaceae significantly decreased.

\section{Correlation Analysis}

Correlation analysis was carried out within the 158 most abundant OTUs and between them and the concentration of ammonium, indole, and $p$-cresol. Correlation analysis among the OTUs did not identify evident clusters (Supplementary Datasheet S2). However, the Bacteroidetes were generally in positive correlation with each other and in negative correlation with a variety of Firmicutes, including taxa that presented different responses to proteins (e.g., Lachnoclostridium and Subdoligranulum). Restricting the analysis to the OTUs that positively responded to proteinaceous substrates confirmed the predominance of different biomarkers in $\mathrm{C}$ and $\mathrm{D}$ cultures (Figure 5). In fact, positive correlations were observed within the biomarkers of grown D (Collinsella, EsherichiaShigella, and Escherichia coli) or C cultures (Anaeortruncus, Oscillibacter, and Sellimonas), whereas negative correlations occurred between $\mathrm{C}$ and $\mathrm{D}$ biomarkers. The putatively proteolytic OTUs of Lachnospiraceae and Ruminococcaceae were generally in positive correlation with each other, with only a few cases of negative correlations. Some negative correlations were also observed between putative proteolytic Firmicutes and Enterobacteria, mainly Sutterella, Parasutterella, and EscherichiaShigella. Sutterella and Parasutterella were mutually exclusive in the samples inoculated with different microbiota.

Positive correlations with ammonium, indole, and/or $p$-cresol, were observed for OTUs attributed to Ruminococcaceae
(13 OTUs), Lachnospiraceae (11 OTUs), Family XIII Clostridiales (2 OTUs), and Eggerthellaceae, Akkermasia, and Parasutterella (1 OTU each) (Figure 6). Some of them were previously identified as growth biomarkers by LEfSe (e.g., Anaerotruncus, Oscillibacter, Dorea, Sellimonas) or presented a positive response to proteins in some cultures (e.g., Akkermasia, and Parasutterella). Positive correlation with metabolites was also detected for some taxa that were not previously identified, such as some Lachnispiraceae (Hungatella and Ruminococcus torques, etc.), Ruminococcaceae (Anaerophilum, Flavonifractor, Intestinimonas, Negativibacillus, Ruminiclostridium, etc.), Family XIII Clostridiales, and Eggerthellaceae. On the other hand, the OTUs of Proteobacteria that became enriched during cultivation did not exhibit any significant correlation with ammonia, indole, or $p$-cresol, with the exception of Parasutterella, which positively correlated with indole.

None of the OTUs that significantly decreased presented any positive correlation with ammonium, indole, or $p$-cresol. On the other hand, many taxa presenting a significant negative response to proteinaceous substrates negatively correlated with ammonium and indole. This is the case with Barnesiellaceae (such as Barnesiella and Coprobacter), Marinifilaceae (including the genus Odoribacter), and several OTUs of Lachnospiraceae and Ruminococcaceae, such as Roseburia, Ruminococcus.

\section{DISCUSSION}

The occurrence of protein catabolism by the gut microbiota was established by early culture-based works that identified Bacteroides and Propionibacterium as dominant proteolytic species and recognized proteolysis as common amongst clostridia, streptococci, staphylococci, and Bacillus species (Macfarlane et al., 1986; Smith and Macfarlane, 1998; Davila et al., 2013). However, proteolysis is a wide concept that can refer to the hydrolysis of proteins to provide microorganisms with a nitrogen source or to proper fermentation of amino acids to obtain energy and C-precursors. The bacterial utilization of proteins involves a complex metabolic network starting with the hydrolysis to peptides and amino acids, which can be assimilated in anabolic processes and/or fermented to gain energy and C-sources.

The gut microbiota enrichment cultures herein described aimed to identify, through a $16 \mathrm{~S}$ rRNA gene survey, the bacterial taxa that take advantage of proteins, peptones, and amino acids as the sole available carbon sources. The approach relied on registering differences in relative bacterial amounts during $\mathrm{pH}$-controlled batch processes, with the awareness that a major increase in some groups may conceal a lesser increase in others. Based on the accumulation of organic acids, ammonia, and indole, the cultures successfully selected bacteria performing protein breakdown and fermentation. In the complex relationship of nutritional cross-feeding interactions that characterizes the gut microbiota, true protein and amino acid fermenters became enriched, especially in the first hours of the process, likely accompanied or followed by bacteria utilizing the fermentation products. 

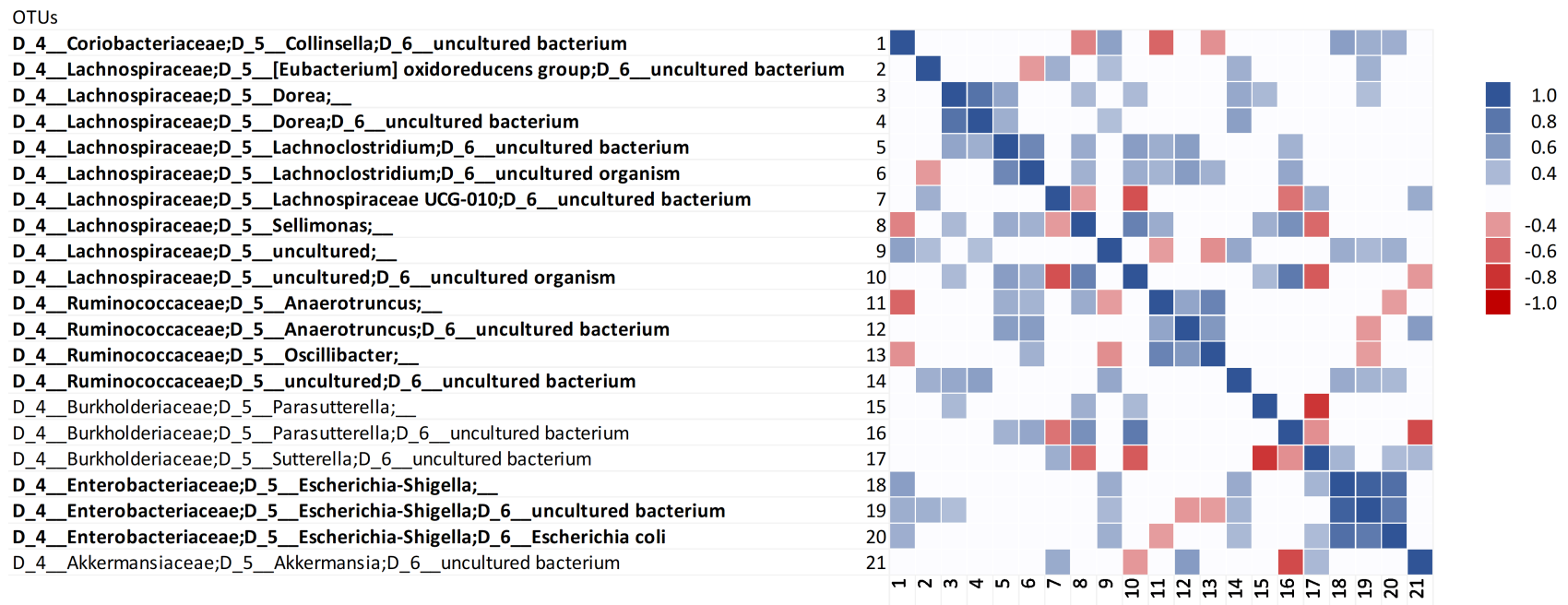

FIGURE 5 | Spearman's rank correlation among the OTUs that positively responded to proteinaceous substrates. Rho values are reported in the heatmap if statistically significant $(P<0.05)$, ranging from the deepest red $(-1)$ to the deepest blue $(+1)$. The OTUs that were evidenced by LEfSe as biomarkers with significant increase are in bold.

Despite differences in the founding communities, the cultures described similar trajectories in the PCo space of Weighted Normalized Unifrac, suggesting that they evolved in the same direction and shared enrichment in phylogenetically similar bacterial groups. Analysis of differential abundances among time-points leads to the identification of taxa participating in protein breakdown and fermentation, particularly within the Enterobacteriaceae, Burkholderiaceae, Ruminococcaceae, and Lachnospiraceae. Other groups, some characterized by saccharolytic metabolism, clearly decreased in relative abundance, for example many Firmicutes (e.g., Roseburia, Ruminococcus, Lachnospira, Dialister, and Erysipelotrichaceae) and Bacteroidetes (e.g., Barnesiellaceae, Prevotellaceae, and Rickenelliaceae).

Despite D cultures being carried out to detect any growth even in the most concentrated taxa, no significant changes in the relative abundance of Bacteroidaceae and related taxa were observed. Bacteroides are major primary degraders of oligo- and polysaccharides (Wexler, 2007). However, members of this genus also produce extracellular proteases and exopeptidases with a variety of arylamidase activities (Riepe et al., 1980; Macfarlane and Macfarlane, 1997; Song et al., 2004), ferment amino acids (Smith and Macfarlane, 1998), and positively correlate with the consumption of protein in human and animal studies (Wu et al., 2011; Rist et al., 2014). In the present study, a wide number of ASVs attributed to Bacteroides presented opposite contributions along the principal coordinates. It is plausible that different populations of Bacteroides, which could not be discriminated into species by the partial $16 \mathrm{~S}$ rRNA gene sequencing, behaved differently with respect to protein breakdown, exerting contrary effects and balancing the counts of the genus. As a whole, by virtue of the great abundance in the gut, Bacteroides likely maintain a pivotal role as primary protein hydrolyzers, making amino acids and peptides available for both biosynthesis reactions and catabolism by a larger number of bacteria, ranging from facultative saccharolytic bacteria to obligate amino acid fermenters.

Because of their easy isolation and cultivation, Enterobacteriaceae such as E. coli, Enterobacter, Klebsiella, and Shigella spp. were among the first gut bacteria to be identified as protein degraders, in agreement with the results of this study, which identified all the taxonomic levels of Proteobacteria as biomarkers of growth on proteins. Enterobacteriaceae, and in particular E. coli, are pathobionts bearing a number of virulence genes that may turn from commensal organisms into pathogens responsible for severe diseases when specific genetic or environmental conditions are altered in the host (Raimondi et al., 2019). High levels of $E$. coli have been associated with inflammatory bowel disease and cancer (Veziant et al., 2016), and thus the proliferation of E. coli and Enterobacteriaceae may promote inflammation, determine colitogenic effects, and favor the growth of subdominant populations playing a role in carcinogenesis.

Correlation analysis pointed out members of Ruminococcaceae and Lachnospiraceae as main producers of the harmful metabolites ammonium, indole, and p-cresol, with several of these OTUs ascribed to uncultured bacteria or to genera not investigated in terms of metabolism, physiology, and relationship with the host. Interestingly, Enterobacteriaceae and E. coli, proteolytic bacteria regarded as major indole producers (Lee and Lee, 2010; Dai et al., 2011), did not present significant correlations with this metabolite or with ammonium and $p$-cresol. As a matter of fact, all of these metabolites reached higher concentrations in C cultures, whereas several taxa of Proteobacteria, including E. coli, bloomed mainly in D cultures, where protein metabolites were less concentrated. 


\section{OTUs}

D_3_Coriobacteriales;D_4_Eggerthellaceae;_;

D_4_Barnesiellaceae;D_5_Barnesiella;D_6_uncultured bacterium

D_4_Barnesiellaceae;D_5_Coprobacter;D_6_uncultured organism

D_4_Marinifilaceae;D_5_Odoribacter;

D_4_Rikenellaceae;D_5_Alistipes;D_6_uncultured bacterium

D_4_Tannerellaceae;D_5_Parabacteroides;

D_4_Tannerellaceae;D_5_Parabacteroides;D_6_uncultured organism

D_4_Streptococcaceae;D_5_Streptococcus;D_6_uncultured bacterium

D_4_Family XIII;D_5_Family XIII AD3011 group;

D_4_Family XIII;D_5_Family XIII AD3011 group;D_6_uncultured bacterium

D_4_Family XIII;D_5_Family XIII UCG-001;D_6_uncultured bacterium

D_4_Lachnospiraceae;D_5_[Eubacterium] xylanophilum group;D_6_uncultured bacterium

D_4_Lachnospiraceae;D_5_[Ruminococcus] torques group;

D_4_Lachnospiraceae;D_5_Dorea;

D_4_Lachnospiraceae;D_5_GCA-900066575;

D_4_Lachnospiraceae;D_5_GCA-900066755; _

D_4_Lachnospiraceae;D_5_Hungatella;

D_4_Lachnospiraceae;D_5_Lachnoclostridium;D_6_uncultured bacterium

D_4_Lachnospiraceae;D_5_Lachnospiraceae FCS020 group;

D_4_Lachnospiraceae;D_5_Lachnospiraceae NC2004 group;D_6_uncultured bacterium

D_4_Lachnospiraceae;D_5_Lachnospiraceae UCG-004;

D_4_Lachnospiraceae;D_5_Lachnospiraceae UCG-008;D_6_uncultured bacterium

D_4_Lachnospiraceae;D_5_Roseburia;D_6_uncultured bacterium

D_4_Lachnospiraceae;D_5_Sellimonas;

D_4_Lachnospiraceae;D_5_uncultured;

D_4_Lachnospiraceae;D_5_uncultured;D_6_uncultured organism

D_4_Ruminococcaceae; ;

D_4_Ruminococcaceae;D_5_Anaerofilum;_

D_4_Ruminococcaceae;D_5_Anaerotruncus;

D_4_Ruminococcaceae;D_5_Faecalibacterium;D_6_uncultured bacterium

D_4_Ruminococcaceae;D_5_Flavonifractor;

D_4_Ruminococcaceae;D_5_Intestinimonas;

D_4_Ruminococcaceae;D_5_Negativibacillus;D_6_uncultured bacterium

D_4_Ruminococcaceae;D_5_Oscillibacter;

D_4_Ruminococcaceae;D_5_Ruminiclostridium 9;D_6_uncultured bacterium

D_4_Ruminococcaceae;D_5_Ruminococcaceae UCG-002;D_6_uncultured bacterium

D_4_Ruminococcaceae;D_5_Ruminococcaceae UCG-003;

D_4_Ruminococcaceae;D_5_Ruminococcaceae UCG-003;D_6_uncultured bacterium

D_4_Ruminococcaceae;D_5_Ruminococcaceae UCG-004;D_6_uncultured bacterium

D_4_Ruminococcaceae;D_5_Ruminococcaceae UCG-013;D_6_uncultured bacterium

D_4_Ruminococcaceae;D_5_Ruminococcus 1;D_6_uncultured bacterium

D_4_Ruminococcaceae;D_5_Ruminococcus 2;D_6_uncultured bacterium

D_4_Ruminococcaceae;D_5_uncultured;

D_4_Ruminococcaceae;D_5_uncultured;D_6_uncultured bacterium

D_4_Erysipelotrichaceae;D_5_Erysipelotrichaceae UCG-003;D_6_uncultured bacterium

D_4_Veillonellaceae;D_5_Veillonella;D_6_uncultured bacterium

D_4_Burkholderiaceae;D_5_Parasutterella;D_6_uncultured bacterium

D_4_Akkermansiaceae;D_5_Akkermansia;D_6_uncultured bacterium

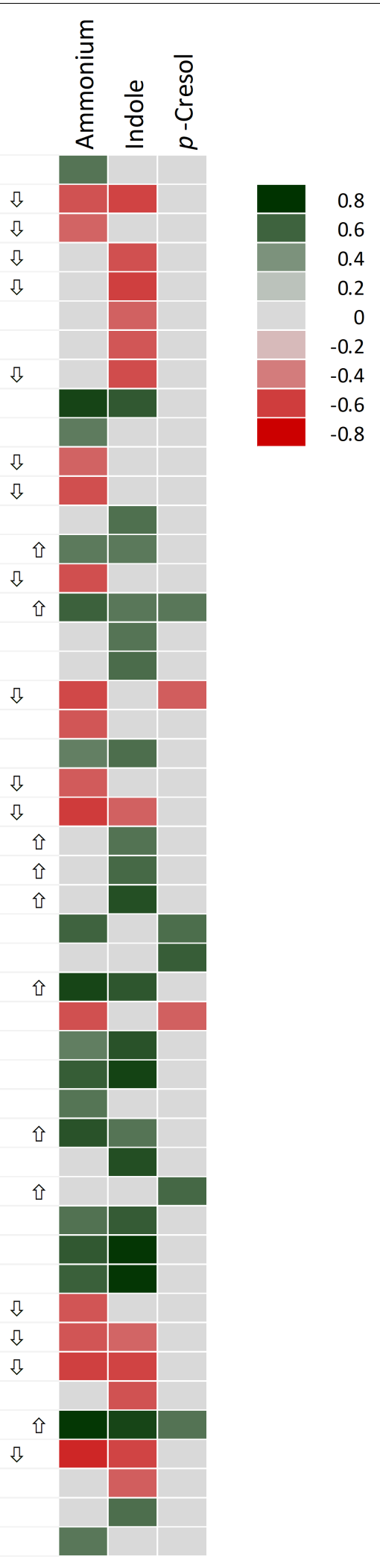

FIGURE 6 | Spearman's rank correlation among the OTUs and ammonium, indole, and p-cresol. Only OTUs significantly correlating with at least one metabolite are displayed. Rho values are reported in the heatmap if statistically significant $(P<0.05)$, ranging from the deepest red $(-1)$ to the deepest green $(+1)$. The OTUs that were evidenced by LEfSe as biomarkers with significant increase $(\widehat{)})$ or decrease $(\sqrt{ })$ are in bold. 
Burkholderiaceae and Desulfovibrionaceae also responded positively to proteinaceous substrates, with an increase of asaccharolytic anaerobes/microaerophiles such as Sutterella, Parasutterella, and Bilophila (Wexler et al., 1996; Ju et al., 2019). These genera are core components of the human gut microbiota, occurring ubiquitously, but are still understudied in terms of physiology and role in the intestinal ecosystem. The present study demonstrated that they take advantage of the breakdown of proteinaceous substrates, providing one of the first insights into their role in the colonic ecology. However, it remains to be investigated whether these asaccharolytic bacteria grew as true amino acid fermenters or utilized the organic acids released by other bacteria (da Silva et al., 2008).

Several other taxa, which are still poorly characterized at the physiological level, emerged as participating in protein catabolism, for some species representing one of the first sources of functional information. Many bacterial biomarkers herein identified are members of Lachnospiraceae, Ruminococcaceae, and Peptostreptococcaceae (such as Anaerotruncus, Dorea, Oscillibacter, Eubacterium oxidoreducens, Lachnoclostridium, Paeniclostridium, Rombutsia, and other unclassified members), for which no information is available or very little is known on their possible role in protein catabolism and amino acid fermentation, apart from some correlation with a protein-rich diet (Taras et al., 2002; David et al., 2014; La Reau and Suen, 2018).

On the other hand, the opportunistic pathogen Acidaminococcus was already known as a true amino acid fermenter that is capable of utilizing amino acids as its sole carbon/energy source (Kim et al., 2004; D’Auria et al., 2011). Acidaminococcus, rarely detected in the founding microbiota, was particularly abundant and grew only in the cultures inoculated with a single founding microbiota.

Similar behavior was observed for the mucus degrader Akkermansia, which was negligible in most cultures but increased abundantly in the cultures of a sole subject. A. mucinifila is claimed as a next-generation probiotic because of the protective effect exerted against metabolic disorders and inflammatory diseases (Cani and de Vos, 2017). It is plausible that proteases are involved in the degradation of mucin and may have supported the growth in the protein-based microbiota cultures.

\section{CONCLUSION}

This study shed light on the bacterial taxa thriving in cultures of human microbiota and utilizing proteins and peptides as their primary substrate for growth. It provided the first information on some enigmatic ubiquitous commensal bacteria. Protein catabolism is claimed to be detrimental for human health on the basis of metabolites like indoles and phenols. In terms of bacterial population, Proteobacteria, including Enterobacteriaceae and E. coli, are among the microbial group that most benefit from protein as C-sources, increasing the risk of the flourishing of opportunistic pathogens or bacteria associated to gut inflammation. However, Lachnospiraceae and Ruminococcaceae, that encompass a number of health-promoting saccharolytic bacteria such as the butyrate producers, presented several OTUs of proteolytic bacteria and showed the strongest correlation with the production of ammonium, indole, and $p$-cresol.

\section{DATA AVAILABILITY STATEMENT}

16S rRNA gene sequences have been submitted to NCBI repository with the BioProject ID: PRJNA540787.

\section{ETHICS STATEMENT}

This study was carried out in accordance with the recommendations of the protocol approved with ref. no. 125-15 by Comitato Etico Provinciale, Azienda Policlinico di Modena, with written informed consent from all subjects. All subjects gave written informed consent in accordance with the Declaration of Helsinki. The protocol was approved by Comitato Etico Provinciale, Azienda Policlinico di Modena, Italy.

\section{AUTHOR CONTRIBUTIONS}

AA, AM, and MR conceived and designed the experiments. CG, MS, SR, and LR carried out the fermentations and chemical analysis. AA, VP-B, RG-L, and AM performed the metagenomic study and the bioinformatics. AA and MR wrote the manuscript with contributions from all other authors.

\section{FUNDING}

This work was funded by FAR 2014 (Fondo di Ateneo per la Ricerca, University of Modena and Reggio Emilia) in the framework of a project entitled "Caratterizzazione della Componente Proteolitica del Microbiota Intestinale Umano" "Characterization of the Proteolytic Component of the Human Gut Microbiota." This work was also supported by grants to AM from the Spanish Ministry of Economy and Competitiveness (project SAF2015-65878-R), Generalitat Valenciana (project Prometeo/2018/A/133), and Asociación Española Contra el Cancer (project AECC 2017-1485), and was co-financed by the European Regional Development Fund (ERDF).

\section{SUPPLEMENTARY MATERIAL}

The Supplementary Material for this article can be found online at: https://www.frontiersin.org/articles/10.3389/fmicb. 2019.02614/full\#supplementary-material 


\section{REFERENCES}

Amaretti, A., Bernardi, T., Leonardi, A., Raimondi, S., Zanoni, S., and Rossi, M. (2013). Fermentation of xylo-oligosaccharides by Bifidobacterium adolescentis DSMZ 18350: kinetics, metabolism, and $\beta$-xylosidase activities. Appl. Microbiol. Biotechnol. 97, 3109-3117. doi: 10.1007/s00253-012-4509-y

Barker, H. A. (1981). Amino acid degradation by anaerobic bacteria. Annu. Rev. Biochem. 50, 23-40. doi: 10.1146/annurev.bi.50.070181.000323

Barrios, C., Beaumont, M., Pallister, T., Villar, J., Goodrich, J. K., Clark, A., et al. (2015). Gut-microbiota-metabolite axis in early renal function decline. PLoS One 10:e0134311. doi: 10.1371/journal.pone.0134311

Blachier, F., Davila, A. M., Mimoun, S., Benetti, P. H., Atanasiu, C., Andriamihaja, M., et al. (2010). Luminal sulfide and large intestine mucosa: friend or foe? Amino Acids 39, 335-347. doi: 10.1007/s00726-018-2558-y

Bolyen, E., Rideout, J. R., Chase, J., Pitman, T. A., Shiffer, A., Mercurio, W., et al. (2018). An introduction to applied bioinformatics: a free, open, and interactive text. J. Open Source Educ. 1:27. doi: 10.21105/jose.00027

Bottari, B., Quartieri, A., Prandi, B., Raimondi, S., Leonardi, A., Rossi, M., et al. (2017). Characterization of the peptide fraction from digested parmigiano reggiano cheese and its effect on growth of lactobacilli and bifidobacteria. Int. J. Food Microbiol. 255, 32-41. doi: 10.1016/j.ijfoodmicro.2017.05.015

Cani, P. D., and de Vos, W. M. (2017). Next-generation beneficial microbes: the case of Akkermansia muciniphila. Front. Microbiol. 8:1765. doi: 10.3389/fmicb. 2017.01765

Cummings, J. H., and Macfarlane, G. T. (1991). The control and consequences of bacterial fermentation in the human colon. J. Appl. Bacteriol. 70, 443-459. doi: 10.1111/j.1365-2672.1991.tb02739.x

da Silva, S. M., Venceslau, S. S., Fernandes, C. L., Valente, F. M., and Pereira, I. A. (2008). Hydrogen as an energy source for the human pathogen Bilophila wadsworthia. Antonie Van Leeuwenhoek 93, 381-390. doi: 10.1007/s10482-0079215-x

Dai, Z. L., Wu, G., and Zhu, W. Y. (2011). Amino acid metabolism in intestinal bacteria: links between gut ecology and host health. Front. Biosci. 16:1768. doi: $10.2741 / 3820$

D’Auria, G., Galán, J. C., Rodríguez-Alcayna, M., Moya, A., Baquero, F., and Latorre, A. (2011). Complete genome sequence of Acidaminococcus intestini RYC-MR95, a gram-negative bacterium from the phylum firmicutes. J. Bacteriol. 193, 7008-7009. doi: 10.1128/JB.06301-11

David, L. A., Maurice, C. F., Carmody, R. N., Gootenberg, D. B., Button, J. E., Wolfe, B. E., et al. (2014). Diet rapidly and reproducibly alters the human gut microbiome. Nature 505, 559-563. doi: 10.1038/nature 12820

Davila, A. M., Blachier, F., Gotteland, M., Andriamihaja, M., Benetti, P. H., Sanz, Y., et al. (2013). Intestinal luminal nitrogen metabolism: role of the gut microbiota and consequences for the host. Pharmacol. Res. 68, 95-107. doi: 10.1016/j.phrs. 2012.11.005

Gänzle, M. G., and Follador, R. (2012). Metabolism of oligosaccharides and starch in lactobacilli: a review. Front. Microbiol. 3:340. doi: 10.3389/fmicb.2012.00340

Ju, T., Kong, J. Y., Stothard, P., and Willing, B. P. (2019). Defining the role of Parasutterella, a previously uncharacterized member of the core gut microbiota. ISME J. 13, 1520-1534. doi: 10.1038/s41396-019-0364-5

Kim, J., Hetzel, M., Boiangiu, C. D., and Buckel, W. (2004). Dehydration of (R)-2hydroxyacyl-CoA to enoyl-CoA in the fermentation of alpha-amino acids by anaerobic bacteria. FEMS Microbiol. Rev. 28, 455-468. doi: 10.1016/j.femsre. 2004.03.001

Kobayashi, J. (2017). Effect of diet and gut environment on the gastrointestinal formation of N-nitroso compounds: a review. Nitric Oxide 73, 66-73. doi: 10.1016/j.niox.2017.06.001

La Reau, A. J., and Suen, G. (2018). The ruminococci: key symbionts of the gut ecosystem. J. Microbiol. 56, 199-208. doi: 10.1007/s12275-018-8024-8024

Lee, J. H., and Lee, J. (2010). Indole as an intercellular signal in microbial communities. FEMS Microbiol. Rev. 34, 426-444. doi: 10.1111/j.1574-6976. 2009.00204.x

Ma, N., Tian, Y., Wu, Y., and Ma, X. (2017). Contributions of the interaction between dietary protein and gut microbiota to intestinal health. Curr. Protein Pept. Sci. 18, 795-808. doi: 10.2174/1389203718666170216153505

Macfarlane, G. T., Cummings, J. H., and Allison, C. (1986). Protein degradation by human intestinal bacteria. J. Gen. Microbiol. 132, 1647-1656. doi: 10.1099/ 00221287-132-6-1647

Macfarlane, S., and Macfarlane, G. T. (1997). Formation of a dipeptidyl arylamidase by Bacteroides splanchnicus NCTC 10825 with specificities towards glycylprolyl-x and valylalanine-x substrates. J. Med. Microbiol. 46, 547-555. doi: 10.1099/00222615-46-7-547

Moore-Connors, J. M., Dunn, K. A., Bielawski, J. P., and Van Limbergen, J. (2016). Novel Strategies for Applied Metagenomics. Inflamm. Bowel Dis. 22, 709-718. doi: 10.1097/MIB.0000000000000717

Raimondi, S., Righini, L., Candeliere, F., Musmeci, E., Bonvicini, F., Gentilomi, G., et al. (2019). Antibiotic resistance, virulence factors, phenotyping, and genotyping of E. coli Isolated from the Feces of Healthy Subjects. Microorganisms 7:E251. doi: 10.3390/microorganisms7080251

Riedel, T., Wetzel, D., Hofmann, J. D., Plorin, S. P. E. O., Dannheim, H., Berges, M., et al. (2017). High metabolic versatility of different toxigenic and nontoxigenic Clostridioides difficile isolates. Int. J. Med. Microbiol. 307, 311-320. doi: 10.1016/j.ijmm.2017.05.007

Riepe, S. P., Goldstein, J., and Alpers, D. H. (1980). Effect of secreted Bacteroides proteases on human intestinal brush border hydrolases. J. Clin. Investig. 66, 314-322. doi: 10.1172/JCI109859

Rist, V. T., Weiss, E., Sauer, N., Mosenthin, R., and Eklund, M. (2014). Effect of dietary protein supply originating from soybean meal or casein on the intestinal microbiota of piglets. Anaerobe 25, 72-79. doi: 10.1016/j.anaerobe.2013. 10.003

Russell, W. R., Duncan, S. H., Scobbie, L., Duncan, G., Cantlay, L., Calder, A. G., et al. (2013a). Major phenylpropanoid-derived metabolites in the human gut can arise from microbial fermentation of protein. Mol. Nutr. Food Res. 57, 523-535. doi: 10.1002/mnfr.201200594

Russell, W. R., Hoyles, L., Flint, H. J., and Dumas, M. E. (2013b). Colonic bacterial metabolites and human health. Curr. Opin. Microbiol. 16, 246-254. doi: 10. 1016/j.mib.2013.07.002

Schloss, P. D., Westcott, S. L., Ryabin, T., Hall, J. R., Hartmann, M., Hollister, E. B., et al. (2009). Introducing mothur: open-source, platform-independent, community-supported software for describing and comparing microbial communities. Appl. Environ. Microbiol. 75, 7537-7541. doi: 10.1128/AEM. 01541- 1549

Scott, K. P., Gratz, S. W., Sheridan, P. O., Flint, H. J., and Duncan, S. H. (2013). The influence of diet on the gut microbiota. Pharmacol. Res. 69, 52-60. doi: 10.1016/j.phrs.2012.10.020

Segata, N., Izard, J., Waldron, L., Gevers, D., Miropolsky, L., Garrett, W. S. et al. (2011). Metagenomic biomarker discovery and explanation. Genome Biol. 12:R60. doi: 10.1186/gb-2011-12-6-r60

Sivaprakasam, S., Prasad, P. D., and Singh, N. (2016). Benefits of short-chain fatty acids and their receptors in inflammation and carcinogenesis. Pharmacol. Ther. 164, 144-151. doi: 10.1016/j.pharmthera.2016.04.007

Smith, E. A., and Macfarlane, G. T. (1996). Enumeration of human colonic bacteria producing phenolic and indolic compounds: effects of $\mathrm{pH}$, carbohydrate availability and retention time on dissimilatory aromatic amino acid metabolism. J. Appl. Bacteriol. 81, 288-302. doi: 10.1111/j.1365-2672.1996. tb04331.x

Smith, E. A., and Macfarlane, G. T. (1998). Enumeration of amino acid fermenting bacteria in the human large intestine: effects of $\mathrm{pH}$ and starch on peptide metabolism and dissimilation of amino acids. FEMS Microbiol. Ecol. 25, 355368. doi: 10.1111/j.1574-6941.1998.tb00487.x

Song, Y. L., Liu, C. X., McTeague, M., and Finegold, S. M. (2004). “Bacteroides nordii" sp. nov. and "Bacteroides salyersae" sp. nov. isolated from clinical specimens of human intestinal origin. J. Clin. Microbiol. 42, 5565-5570. doi: 10.1128/JCM.42.12.5565-5570.2004

Taras, D., Simmering, R., Collins, M. D., Lawson, P. A., and Blaut, M. (2002). Reclassification of Eubacterium formicigenerans Holdeman and Moore 1974 as Dorea formicigenerans gen. nov., comb. nov., and description of Dorea longicatena sp. nov., isolated from human faeces. Int. J. Syst. Evol. Microbiol. 52, 423-428. doi: 10.1099/00207713-52-2-423

Veziant, J., Gagnière, J., Jouberton, E., Bonnin, V., Sauvanet, P., Pezet, D., et al. (2016). Association of colorectal cancer with pathogenic Escherichia coli: focus on mechanisms using optical imaging. World J. Clin. Oncol. 7, 293-301. doi: 10.5306/wjco.v7.i3.293

Walker, A. W., Duncan, S. H., McWilliam Leitch, E. C., Child, M. W., and Flint, H. J. (2005). pH and peptide supply can radically alter bacterial populations and short-chain fatty acid ratios within microbial communities from the human colon. Appl. Environ. Microbiol. 71, 3692-3700. doi: 10.1128/AEM.71.7.36923700.2005

Wexler, H. M. (2007). Bacteroides: the good, the bad, and the nitty-gritty. Clin. Microbiol. Rev. 20, 593-621. doi: 10.1128/cmr.00008-07 
Wexler, H. M., Reeves, D., Summanen, P. H., Molitoris, E., McTeague, M., Duncan, J., et al. (1996). Sutterella wadsworthensis gen. nov., sp. nov., and bile-resistant microaerophilic Campylobacter gracilis-like clinical isolates. Int. J. Syst. Bacteriol. 46, 252-258. doi: 10.1099/00207713-46-1-252

Wilson, B., and Whelan, K. (2017). Prebiotic inulin-type fructans and galacto-oligosaccharides: definition, specificity, function, and application in gastrointestinal disorders. J. Gastroenterol. Hepatol. 32, 64-68. doi: 10.1111/jgh. 13700

Wu, G. D., Chen, J., Hoffmann, C., Bittinger, K., Che, Y. Y., Keilbaugh, S. A., et al. (2011). Linking long-term dietary patterns with gut microbial enterotypes. Science 334, 105-108. doi: 10.1126/science.1208344
Conflict of Interest: The authors declare that the research was conducted in the absence of any commercial or financial relationships that could be construed as a potential conflict of interest.

Copyright (c) 2019 Amaretti, Gozzoli, Simone, Raimondi, Righini, Pérez-Brocal, Garcia-López, Moya and Rossi. This is an open-access article distributed under the terms of the Creative Commons Attribution License (CC BY). The use, distribution or reproduction in other forums is permitted, provided the original author(s) and the copyright owner(s) are credited and that the original publication in this journal is cited, in accordance with accepted academic practice. No use, distribution or reproduction is permitted which does not comply with these terms. 